\title{
A Practical Review of Functional MRI Anatomy of the Language and Motor Systems
}

\author{
(D) V.B. Hill, (D) C.Z. Cankurtaran, (D)B.P. Liu, (D)T.A. Hijaz, (D) M. Naidich, (D)A.J. Nemeth, (D). Gastala, (D) C. Krumpelman, (D)E.N. McComb, \\ and (D) A.W. Korutz
}

\begin{abstract}
SUMMARY: Functional MR imaging is being performed with increasing frequency in the typical neuroradiology practice; however, many readers of these studies have only a limited knowledge of the functional anatomy of the brain. This text will delineate the locations, anatomic boundaries, and functions of the cortical regions of the brain most commonly encountered in clinical practice-specifically, the regions involved in movement and language.
\end{abstract}

ABBREVIATIONS: FFA $=$ fusiform face area; IPL $=$ inferior parietal lobule; PPC $=$ posterior parietal cortex; SMA $=$ supplementary motor area; VOTC $=$ ventral occipitotemporal cortex

T his article serves as a review of the functional areas of the brain most commonly mapped during presurgical fMRI studies, specifically targeting movement and language. We have compiled what we hope is a useful, easily portable, and concise resource that can be accessible to radiologists everywhere. We begin with a review of the language-processing system. Then we describe the gross anatomic boundaries, organization, and function of each anatomic region.

\section{Dorsal and Ventral Streams in Language and Visual Processing}

Two anatomically and functionally differentiated parallel processing streams serve as a theoretic model for higher order cognitive processes: the ventral and dorsal streams. These concepts of ventral and dorsal streams as well as their functional and anatomic connectivity are introduced here and will be referenced later in this article. The dual-stream model was first conceptualized for vision by Ungerleider and Mishkin in their seminal work, ${ }^{1}$ hypothesizing that dual and distinct computational streams for vision proceeded in parallel, with the dorsal stream

Received November 26, 2018; accepted after revision April 24, 2019.

From the Departments of Radiology (V.B.H., C.Z.C., B.P.L., T.A.H., M.N., A.J.N., J.G., C.K., E.N.M., A.W.K.), Neurology (A.J.N.), and Radiation Oncology (B.P.L.), Northwestern University Feinberg School of Medicine, Chicago, Illinois.

B.P.L. is supported by P50CA221747 SPORE for Translational Approaches to Brain Cancer. A.J.N. is supported by RO1HL092259, SP0039078: Cerebral Autoregulation Monitoring to Reduce Brain Injury from Cardiac Surgery.

Please address correspondence to Virginia B. Hill, MD, Neuroradiology, Northwestern University Feinberg School of Medicine, Suite 1400, 676 N. St. Clair St, Chicago, IL 60611; e-mail virginiahill2@mac.com or virginia.hill@nm.org; @virginiabhill

- Indicates open access to non-subscribers at www.ajnr.org

http://dx.doi.org/10.3174/ajnr.A6089 serving to analyze spatial position and the ventral stream working to identify what an object is. Influenced by the dorsal and ventral stream model of vision, Hickok and Poeppel ${ }^{2}$ hypothesized a similar framework for language. In this model, the ventral stream, or lexical-semantic system, is involved in sound-to-meaning mappings associated with language comprehension and semantic access. A prototype task of the ventral stream is listening to and deriving meaning from speech. ${ }^{3}$ The dorsal stream, or phonological system, is involved in sound-to-motor mappings associated with producing and articulating language. ${ }^{2,3}$ Hence, a prototype task of the dorsal stream is repeating speech. ${ }^{3}$

The dorsal stream of language originates in the peri-Sylvian region within the superior temporal gyrus and superior temporal sulcus and travels through the supramarginal gyrus to the frontal lobe, including the premotor cortex and inferior frontal gyrus. The major fiber tract of the dorsal stream is the superior longitudinal fasciculus/arcuate fasciculus, connecting the classic posterior temporal receptive language area (Wernicke area) with the frontal expressive language area (Broca area), as well as additional extensions to the inferior premotor cortex within the frontal lobe (Fig 1). ${ }^{4-6}$ The model of the Broca and Wernicke language areas devoted to the production and understanding of speech, respectively, has become richer across the years so that now receptive and expressive language understanding is more complex, with dependence of both on larger areas of the network and with substantial individual variation. ${ }^{7}$ Studies have suggested that the superior longitudinal fasciculus/arcuate fasciculus can be separated into 4 subcomponents (superior longitudinal fasciculus I-III) with the arcuate fasciculus composing the fourth subcomponent. ${ }^{8,9}$ Specifically, the superior longitudinal fasciculus III and arcuate fasciculus play a major role in the dorsal language stream. ${ }^{6}$ 


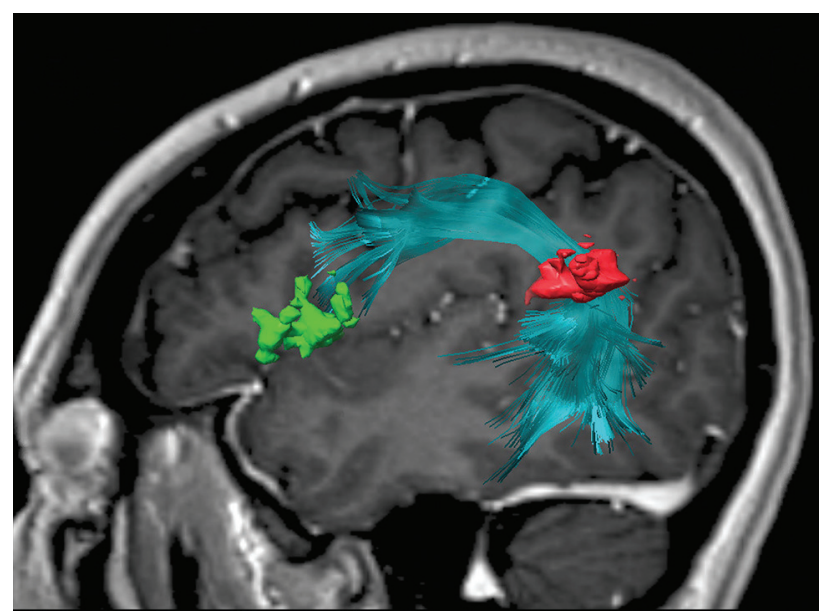

FIG 1. Sagittal 3D-FLAIR image with a superimposed diffusion tensor image of the arcuate fasciculus between the inferior parietal lobule (pars opercularis and pars triangularis) (green) and the angular gyrus (red). The frontotemporal segment of the arcuate fasciculus connects the Broca area at the inferior frontal gyrus with the Wernicke area more posteriorly, traditionally at the posterior superior temporal gyrus. However, this connection is variable, and in this case, it is at the angular gyrus/posterior superior temporal area.

The ventral stream of language involves the middle and inferior temporal gyri, anterior temporal lobe, and ventrolateral prefrontal cortex. ${ }^{10}$ The major tract of the ventral stream is the inferior occipitofrontal fasciculus, with additional contributions by the uncinate fasciculus, extreme capsule, middle longitudinal fasciculus, and inferior longitudinal fasciculus. ${ }^{5,6,10,11}$ While the dorsal stream is left-lateralized, the ventral stream is bilateral. ${ }^{3,12}$ Evidence suggests that while there are some tasks that segregate the ventral and dorsal streams, successful execution requires complex collaboration and seamless integration of processing between the 2 systems. ${ }^{13}$

\section{Primary Motor and Primary Sensory Cortices}

General Anatomic Borders. The primary motor and sensory cortices occupy the pre- and postcentral gyri, respectively (Fig 2A). The precentral gyrus, bounded anteriorly by the precentral sulcus and posteriorly by the central sulcus, extends inferiorly toward the lateral sulcus and becomes contiguous with the inferior aspect of the postcentral gyrus. The superior aspect of the gyrus extends onto the medial surface of the cerebral hemisphere and merges with the anterior paracentral lobule. The postcentral gyrus, bounded anteriorly by the central sulcus and posteriorly by the postcentral sulcus, extends inferiorly and joins the precentral gyrus just above the lateral sulcus. Superiorly, the gyrus passes over the midline of the hemisphere and terminates in the posterior paracentral lobule. ${ }^{14}$

Anatomic Organization. The primary motor cortex does not encompass the entire precentral gyrus but rather tapers inferiorly along the posterior margin of the gyrus. The nonmotor cortex occupies the remainder of the gyrus. ${ }^{15-17}$

The motor and sensory cortices have a well-defined somatotopic organization. In other words, each part of the body can be reliably and predictably mapped to a specific region of the contralateral cortex. Specifically, the cells of the motor and sensory cortices responsible for the lips and face occupy the inferior segments, followed superiorly by the regions for the fingers and hands and a small region for the trunk. The final component, corresponding to the leg and foot, bridges the superior aspect of the gyrus and extends onto the medial surface of the hemisphere. The amount of cortex devoted to any anatomic region is proportional to the degree of precision or discrimination necessary for optimal function of that body part. The resultant distorted homunculus emphasizes the face and lips and the fingers and hands. ${ }^{18,19}$

Function.

Primary Motor Cortex. The primary motor cortex initiates voluntary movement through the corticospinal tract (Fig 2B), which also receives contributions from the premotor cortex, supplementary cortex, and somatosensory cortex. ${ }^{17}$ A large percentage of the corticospinal tract fibers cross in the pyramidal decussation and connect to motor neurons in the contralateral spinal cord to trigger movement. The motor cortex also connects with the cerebellum and brain stem. ${ }^{19}$ Through this network, subcortical regions and other cortical areas influence the input and output of the primary motor cortex. ${ }^{20}$

A lesion in the primary motor cortex, such as an acute infarct, classically results in motor weakness (Fig 3).

Primary Somatosensory Cortex. The primary somatosensory cortex processes tactile and proprioceptive information, receiving fine-touch and proprioception input from the dorsal columnmedial lemniscus pathway and information regarding pain, temperature, and touch from the lateral and ventral spinothalamic tracts. ${ }^{17,19}$ Similar to its motor counterpart, the somatosensory cortex also receives input from other cortical regions, and subcortical areas modify the output from this region. ${ }^{21}$

\section{Supplementary Motor Area}

The supplementary motor area (SMA) and the pre-SMA are discussed separately in this article, with the acknowledgment that their functions are nuanced, their borders are indistinct and functionally variable, and the 2 likely represent a continuum rather than discrete anatomic areas. ${ }^{22}$

General Anatomic Borders. The SMA occupies the dorsomedial aspect of the superior frontal gyrus, immediately anterior to the foot motor representation, and is bounded medially by the interhemispheric falx and laterally by the premotor cortex (Fig $2 A){ }^{23}$ The cingulate sulcus is inferior to the SMA. The anterior margin of the SMA is defined by a perpendicular line through the corpus callosum rostrum. The $\mathrm{V}$ line, a vertical line through the posterior aspect of the anterior commissure, differentiates the rostral from the caudal SMA. ${ }^{24}$

Anatomic Organization. The SMA demonstrates somatotopic organization. From anterior to posterior, the SMA represents the head, trunk, upper extremity, and lower extremity, with some studies showing more detailed somatotopy of the face, neck, and proximal/ distal upper and lower extremities. ${ }^{23}$ While the SMA demonstrates greater activation with tasks performed using the contralateral side of the body, humans do have a dominant SMA that can show bilateral activation. ${ }^{23}$ Word generation and working memory tasks activate the rostral SMA predominantly on the left side; complex motor and sensory tasks activate the contralateral caudal SMA. ${ }^{24}$

AJNR Am J Neuroradiol 40:1084-90 Jul 2019 www.ajnr.org 
Function. The SMA receives input from the motor, premotor, and sensory cortices and provides a wide array of outputs, including those to the primary motor cortex, basal ganglia, thalamus,
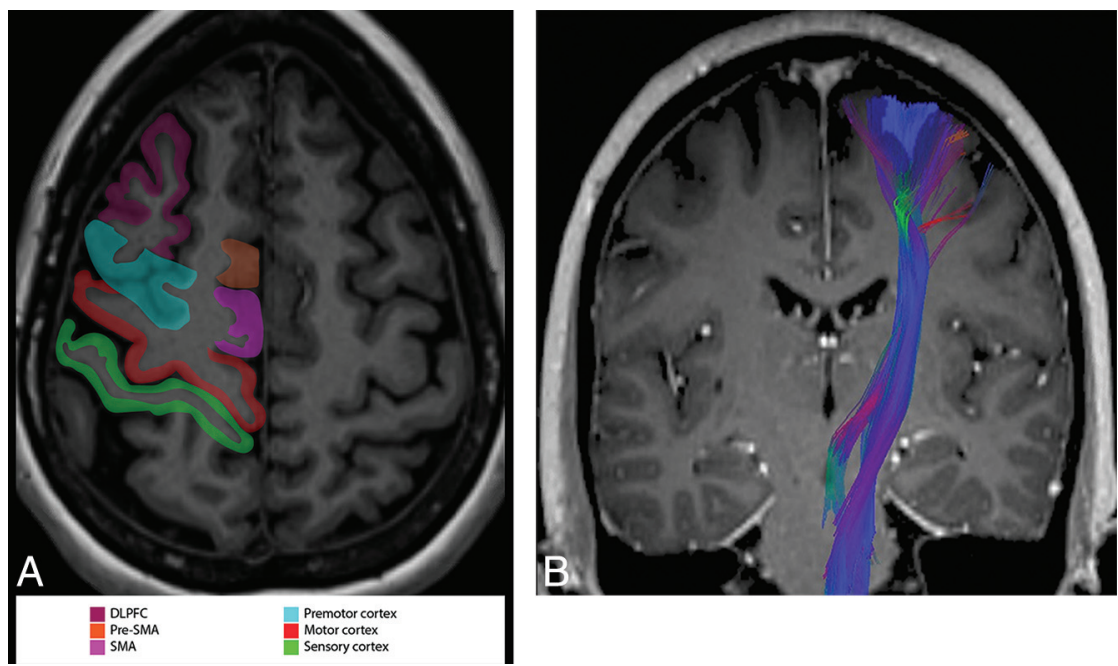

FIG 2. A, Axial MPRAGE image through the sensorimotor cortex with functional areas labeled in the right cerebral hemisphere. $B$, Coronal contrast-enhanced MPRAGE image with superimposed diffusion tensor imaging view of the corticospinal tract. DLPC, dorsolateral prefrontal cortex; SMA, supplementary motor area.
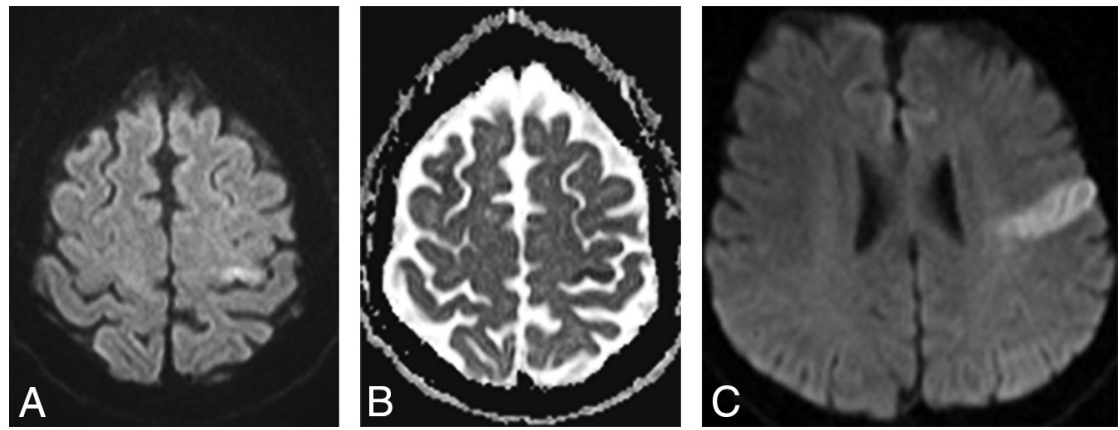

FIG 3. DWI $(A)$ and ADC map $(B)$ demonstrate a left hand/arm motor infarct in a patient with sudden onset of right-arm weakness. C, DWI demonstrates a left facial motor infarct in a patient with facial weakness.
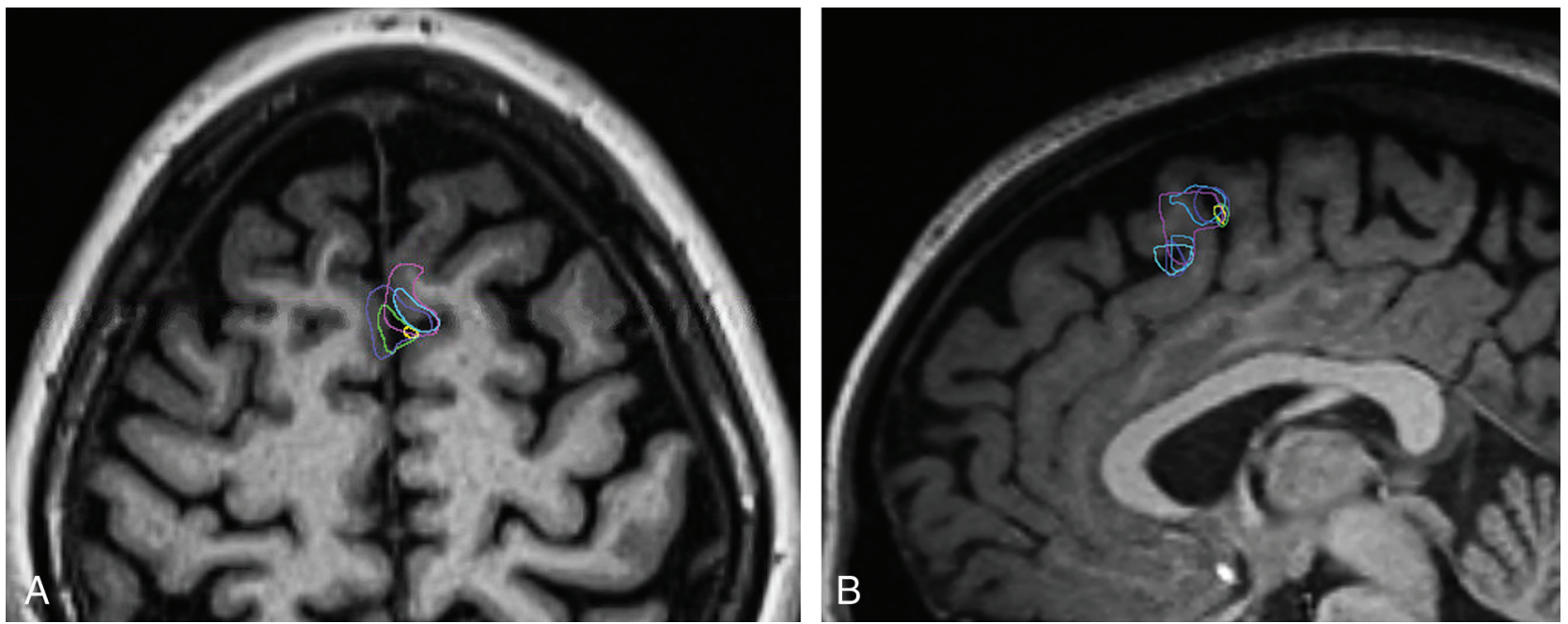

FIG 4. Functional MR imaging blood oxygen level-dependent activation of the SMA superimposed on axial ( $A$ ) and sagittal ( $B$ ) MPRAGE images. Dark blue designates activation during antonym generation; turquoise, during picture naming; purple, during rhyming; light blue, during silent word generation; yellow, during finger movement; and green, during lip movement. proximately one-third of the corticospinal tract. ${ }^{25}$ The SMA is involved in the planning, coordination, and initiation of movement, particularly complex hand motions and action sequences that involve both sides of the body. ${ }^{22-24}$ Learning new motion sequences activates the rostral SMA, but the performance of those sequences activates the caudal SMA. The SMA is also involved in posture, selection of the laterality of a movement, speech (such as word generation and comprehension of the spoken word), working memory, and pain sensation (Fig 4). ${ }^{23,24}$

Lesions of the dominant SMA such as tumors or anterior cerebral artery infarctions result in abulia, gait apraxia, and transient weakness. ${ }^{22}$ Resection of speech (Fig 5). "SMA Syndrome” results from unilateral resection of the SMA and is characterized by contralateral akinesia and mutism, but preservation of extremity muscle strength. ${ }^{26}$ Lesions can also result in "alien limb" syndrome in which spontaneous movements of the limb, such as grasping, are uncontrollable by the patient, often countering the patient's attempted goal-directed motion. ${ }^{22}$ Left middle cerebral artery infarct causing aphasia can prompt compensation by and reorganization of the nondominant SMA also can inhibit speech function to the SMA. ${ }^{24}$ 

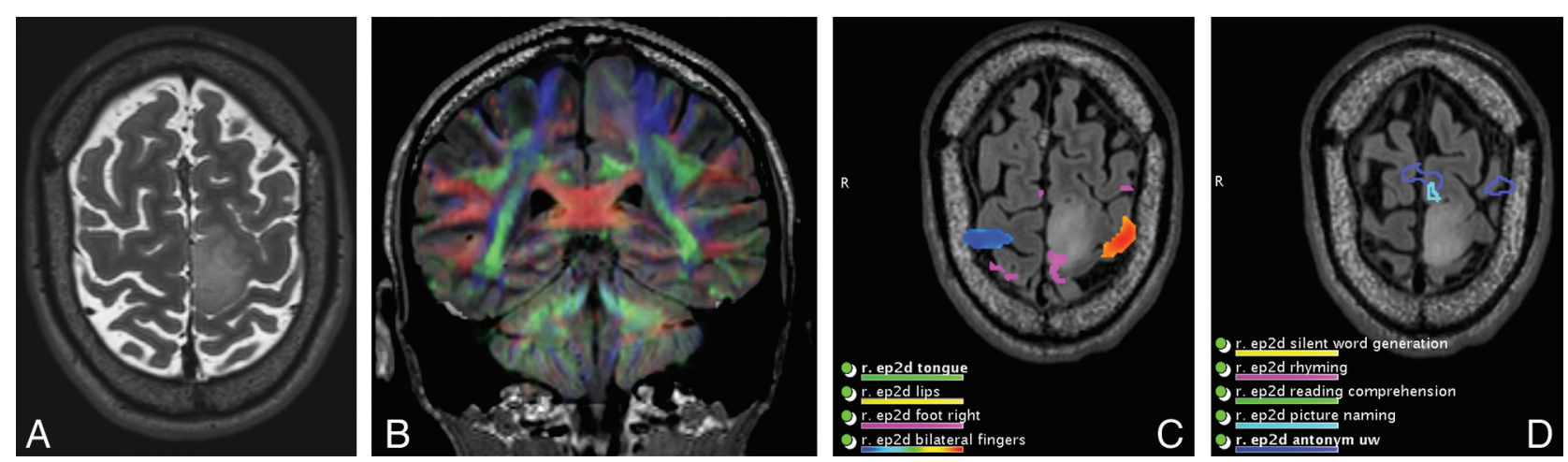

FIG 5. A patient with a left precentral gyrus/paracentral lobule and an SMA mass who initially presented with seizure. A, Axial T2-weighted MR imaging shows a left SMA mass. The patient underwent subtotal resection complicated by transient postoperative right hemiparesis (SMA syndrome). Pathology showed a diffuse infiltrating astrocytoma, isocitrate dehydrogenase-mutant, World Health Organization grade II. DTI superimposed on coronal FLAIR $(B)$ and axial functional MR imaging show right foot $(C)$ and language $(D)$ SMA activation at the margins of the mass.

\section{Presupplementary Motor Area}

General Anatomic Borders. The pre-SMA is located anterior to the SMA within the dorsal aspect of the superior frontal gyrus (Fig $2 A)$. Similar to the SMA, the pre-SMA is bounded medially by the interhemispheric falx and laterally by the premotor cortex. The ventral (inferior) boundary of the pre-SMA is the anterior cingulate motor area. ${ }^{23,24}$

Anatomic Organization. Somatotopy of the pre-SMA is similar to that of the SMA, albeit cruder, with the head, upper extremity, and lower extremity represented from anterior to posterior. ${ }^{23}$

Function. The pre-SMA is activated when learning new sequential movements ${ }^{24}$ and when incorporating visual input into planning internally generated tasks. ${ }^{23}$ In addition, the predominantly left-sided pre-SMA is activated when performing word-generation and working-memory tasks. ${ }^{24}$

\section{Premotor Region Proper}

General Anatomic Borders. The premotor area comprises the dorsal superior frontal gyrus, dorsal middle frontal gyrus, and the anterior bank of the precentral gyrus, ventral to the primary motor area (Fig 2A). ${ }^{23}$

Anatomic Organization. Somatotopic organization of the premotor area is similar to but cruder than that of the primary motor cortex, with medial-to-lateral representations for the leg, trunk, arm, face, and mouth. ${ }^{25}$

Function. The premotor area integrates auditory, visual, and somatosensory stimuli in the selection of movement, initiation of movement, and performance of movement-related tasks. The premotor area attends to externally guided movement, in contradistinction to the SMA and pre-SMA, which are involved in selfinitiated movements. Similar to the SMA, the premotor region also helps determine the laterality of motion. ${ }^{23}$

The premotor cortex contains "mirror neurons," as does the posterior parietal cortex (PPC). These neurons are active when a person performs or watches another perform a series of movements; this observation allows us to tap into our own advanced motor circuits to understand the actions of others.

\section{Posterior Parietal Cortex}

General Anatomic Borders. The PPC is caudal and posterior to the primary somatosensory cortex and postcentral sulcus. The PPC comprises the superior parietal lobule and the inferior parietal lobule (IPL), which are separated by the intraparietal sulcus. The medial portion of the superior parietal lobule is the precuneus, located on the medial surface of each cerebral hemisphere in front of the parietal-occipital sulcus and cranial and anterior to the cuneus. The marginal ramus of the cingulate sulcus separates the paracentral lobule from the precuneus, and the subparietal sulcus separates the precuneus from the posterior cingulate cortex. The subparietal sulcus forms the ventral margin of the superior parietal lobule on the medial surface.

The IPL is further divided into 2 gyri: Caudally, the angular gyrus caps the end of the superior temporal sulcus and is continuous with the middle temporal gyrus; rostrally, the supramarginal gyrus caps the end of the Sylvian fissure (Fig 6A). The supramarginal gyrus is continuous anteriorly with the postcentral gyrus and posteriorly with the superior temporal gyrus. ${ }^{27-29}$

Function. The PPC is known for neural processes underlying attention. The important role of the PPC to attention is perhaps best demonstrated by parietal insults or lesions that result in hemispatial or contralateral neglect. The most common lesion site in patients with hemispatial neglect is the right temporoparietal junction, a part of the ventral attention network, and the adjacent superior temporal gyrus. Damage to the nondominant PPC can result in left hemispatial neglect. ${ }^{27,28,30}$

Left-hemispheric PPC lesions in humans result in apraxia and extinction, demonstrating the role of the PPC in high-order motor functions. Functional imaging studies demonstrate that rostral IPL areas activate during the planning of a motor response or movement execution. The IPL is also activated with grasping, viewing graspable objects, conceptualizing actions, evaluating the motor significance of sensory stimuli, basing decisions on perceptions, and judging and observing actions. The intraparietal sulcus and portions of the superior parietal lobule are involved in visuomotor integration. A major function of the PPC is the maintenance of a spatial reference for goal-directed movements. ${ }^{27,28,31,32}$

AJNR Am J Neuroradiol 40:1084-90 Jul 2019 www.ajnr.org 


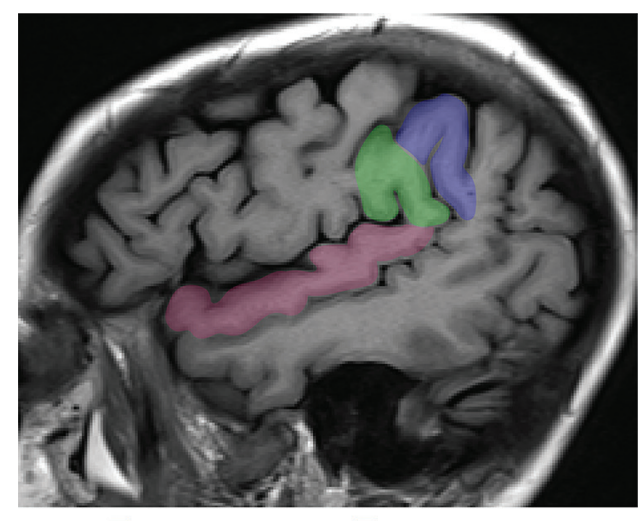

A
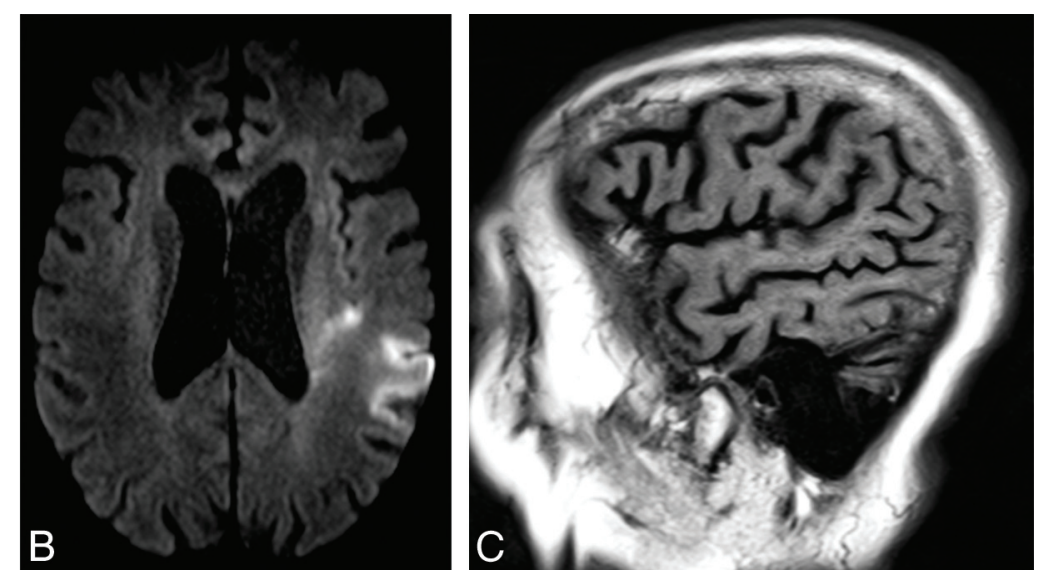

FIG 6. $A$, Sagittal MPRAGE image through the inferior parietal lobule demonstrates the supramarginal gyrus and angular gyrus. The superior temporal gyrus also is highlighted. Axial DWI $(B)$ and sagittal T1-weighted $(C)$ images of a left IPL infarct. The patient presented with the inability to understand and could not produce any comprehensible speech. The IPL is involved in semantic and phonologic processing. Patients with an infarct in this region have severe word-and sentence-comprehension deficits due to a transcortical sensory aphasia. Subdivisions of the arcuate fasciculus connect the IPL to the posterior temporal area as well as the IPL to the Broca area.
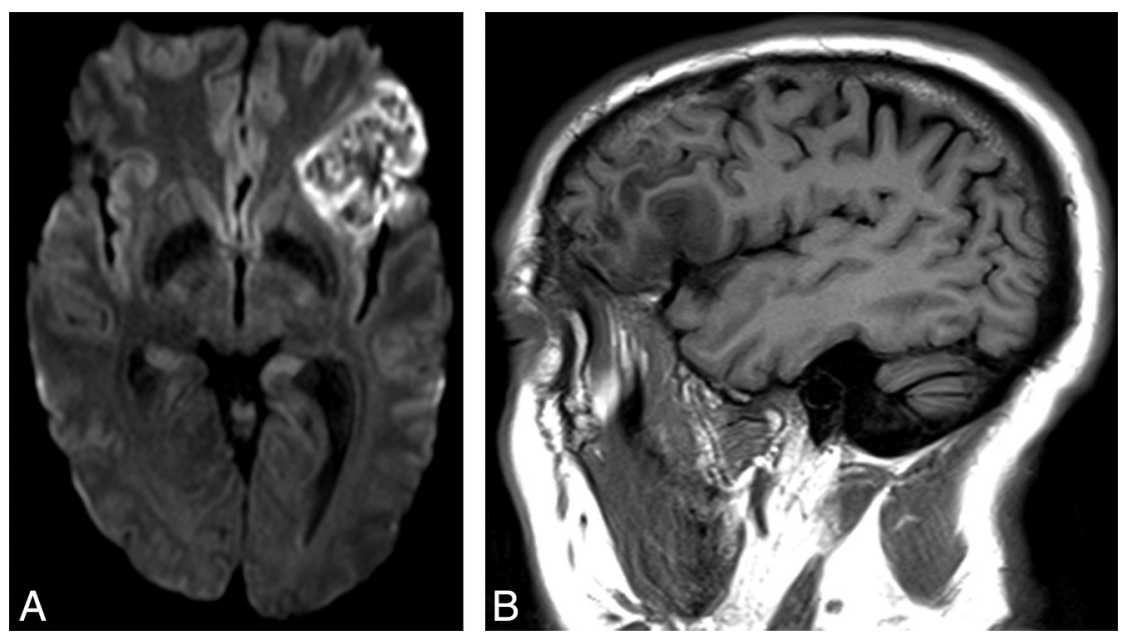

FIG 7. A left Broca infarct with classic expressive aphasia. A, Axial diffusion-weighted image demonstrates restricted diffusion at the classic Broca area. $B$, Sagittal T1-weighted image demonstrates hypointensity due to edema from the infarct.

A language-related area exists within the posterior IPL (Fig 6B,-C), mainly within the angular gyrus. The PPC, particularly the IPL, performs semantic and phonologic processing. The angular gyrus with the adjacent white matter is the most common parietal lesion site in patients with severe word and sentence comprehension deficits due to transcortical sensory aphasia. The PPC is also involved in verbal fluency tasks. ${ }^{27,28,33}$ Studies of white matter connectivity using diffusion-tensor imaging found that subdivisions of the arcuate fasciculus connect the Broca area to the rostral IPL or the IPL to posterior temporal areas; this feature may account for different manifestations of aphasia resulting from damage to different points along the arcuate fasciculus (Fig 1). ${ }^{34,35}$

Arithmetic operations and the processing and understanding of numbers are also functions of the PPC. Injury to the PPC can result in dyscalculia, as is seen in Gerstmann syndrome. Gerstmann syndrome results from damage to the angular gyrus of the language-dominant hemisphere and is characterized by agnosia, agraphia or dysgraphia, dyslexia, right-left disorientation, as well as acalculia or dyscalculia. In some instances, Gerstmann syndrome is also associated with aphasia. ${ }^{27}$

\section{Posterior Superior Temporal Lobe}

General Anatomic Borders. The posterior superior temporal lobe (Fig 6A) includes the posterior two-thirds of the superior temporal gyrus and the cortex extending medially into the posterosuperior aspect of the Sylvian fissure. The superior surface of the superior temporal gyrus, located within the Sylvian fissure, contains 3 distinct areas: the planum polare anteriorly, transverse temporal (Heschl) gyri medially, and the planum temporale posteriorly.

Function. Functional and anatomic studies localize the primary auditory cortex to the transverse temporal (Heschl) gyri and the secondary auditory cortex to the surrounding territory. ${ }^{36,37}$

Damage to projections from the secondary auditory cortex into the posterior Sylvian fissure results in pure word deafness with preserved nonspeech hearing. ${ }^{38,39}$ In the language-dominant hemisphere, the area receiving these projections, area Spt, plays a role in sensorimotor integration as the beginning of the dorsal language stream. Area Spt is located in the Wernicke area (Fig 1); however, damage to area Spt usually results in conductive aphasia. ${ }^{40}$ Recent research questions the exact location of the Wernicke area. Classic receptive aphasia is often associated with lesions outside the traditional boundaries of the Wernicke area. ${ }^{41,42}$

\section{Ventrolateral Prefrontal Cortex}

General Anatomic Borders. The ventrolateral prefrontal cortex is located within the inferior frontal gyrus.

Function. The Broca area is part of a larger network dedicated to language processing and production, most commonly lateralizing to the left hemisphere. Traditionally, the Broca area represents where speech is articulated into meaningful sounds. ${ }^{24}$ Lesion studies suggest a role in internal conflict resolution by selecting 
among competing linguistic representations as part of the cognitive control domain of the lateral prefrontal cortex. ${ }^{43}$ Lesions typically result in motor aphasia with maintained speech comprehension (Fig 7).

\section{Cingulate Cortex}

General Anatomic Borders. The cingulate gyrus, coursing parallel to the corpus callosum, derives its name from the Latin for "belt." The superior frontal gyrus lies anterior and superior to it, and the precuneus abuts it posteriorly.

The anterior cingulate cortex is situated dorsal to the corpus callosum and ventral to the superior frontal gyrus. ${ }^{44}$ The posterior cingulate cortex lies at the medial aspect of the inferior portion of the parietal lobe. Superiorly, it is defined by the marginal ramus of the cingulate sulcus and inferiorly by the corpus callosum. Anteriorly, the posterior cingulate cortex is defined by the midcingulate region and posteriorly by the parieto-occipital sulcus. Along with the precuneus and retrosplenial cortex, it constitutes the posteromedial cortex, and with the retrosplenial cortex, it forms the retrosplenial gyrus. ${ }^{45}$

Function. The midcingulate region contains motor function with corticospinal tract projections. ${ }^{45}$ An fMRI study suggested 3 somatotopically organized motor zones, distinct from SMA activation, in the cingulate/paracingulate region. ${ }^{46}$

\section{Ventral Occipitotemporal Cortex}

General Anatomic Borders. The division between the temporal and occipital lobes is the lateral parietotemporal line, an imaginary boundary extending from the superolateral terminus of the parieto-occipital fissure inferiorly to the preoccipital notch. Several gyri and sulci are continuous between the temporal and occipital lobes across this line; those on the inferior (ventral) aspect of the brain, the lingual gyrus and fusiform gyrus, make up the ventral occipitotemporal cortex (VOTC).

Function. The VOTC forms the path of the ventral visual stream, which maps perceptions of the visual system to conceptual representations. ${ }^{47}$ There is evidence for several areas of functional specialization within the extrastriate ventral stream, each responsible for identifying a particular type of stimulus. Primarily located within the right fusiform gyrus, the fusiform face area (FFA) is responsible for the identification of particular faces. ${ }^{48-50}$ Injury to the FFA can result in prosopagnosia, the inability to recognize familiar faces with the retained ability to identify that a visual stimulus is a face. The corresponding functional unit within the left fusiform gyrus is the visual word form area, ${ }^{51-53}$ injury to which causes pure alexia where a patient cannot read but can speak and comprehend language normally. ${ }^{54}$ Bilateral lesions involving the VOTC can result in agnosia of other types of visual objects, with the retained ability to identify the objects using other sensory inputs. ${ }^{55}$ The lingual gyrus is continuous rostrally with the parahippocampal gyrus and caudally with the superior visual field region of the primary visual cortex. Injuries to the lingual gyri and portions of the fusiform gyri can result in the inability to appreciate color (achromotopsia) and the inability to name colors (color anomia). ${ }^{55}$ The lingual gyrus also may significantly link the visual system and the limbic system, mediating the formation of visual memories and the emotional attachments to images. ${ }^{55,56}$

\section{CONCLUSIONS}

We hope this review of the anatomy and function of the major cortical regions of the brain will foster a better understanding of cortical organization and function.

Disclosures: Virginia B. Hill—UNRELATED: Consultancy: Google.

\section{REFERENCES}

1. Ungerleider LG, Mishkin M. Two cortical visual systems. In: Ingle D, Goodale MA, Mansfield RJ, eds. Analysis of Visual Behavior. Cambridge: MIT Press; 1982:549-86

2. Hickok G, Poeppel D. Dorsal and ventral streams: a framework for understanding aspects of the functional anatomy of language. Cognition 2004;92:67-99 CrossRef Medline

3. Hickok G, Poeppel D. The cortical organization of speech processing. Nat Rev Neurosci 2007;8:393-402 CrossRef Medline

4. Smits M, Jiskoot LC, Papma JM. White matter tracts of speech and language. Semin Ultrasound CT MR 2014;35:504-16 CrossRef Medline

5. Axer $\mathrm{H}$, Klingner CM, Prescher A. Fiber anatomy of dorsal and ventral language streams. Brain Lang 2013;127:192-204 CrossRef Medline

6. Dick AS, Bernal B, Tremblay P. The language connectome: new pathways, new concepts. Neuroscientist 2014;20:453-67 CrossRef Medline

7. Binder JR. fMRI is a valid noninvasive alternative to Wada testing. Epilepsy Behav 2011;20:214-22 CrossRef Medline

8. Yeterian EH, Pandya DN, Tomaiuolo F, et al. The cortical connectivity of the prefrontal cortex in the monkey brain. Cortex 2012;48: 58-81 CrossRef Medline

9. Makris NK, Kennedy DN, McInerney S, et al. Segmentation of subcomponents within the superior longitudinal fasciculus in humans: a quantitative, in vivo, DT-MRI study. Cereb Cortex 2005; 15:854-69 CrossRef Medline

10. Saur D, Kreher BW, Schnell S, et al. Ventral and dorsal pathways for language. Proc Natl Acad Sci U S A 2008;105:18035-40 CrossRef Medline

11. Parker GJ, Luzzi S, Alexander DC, et al. Lateralization of ventral and dorsal auditory-language pathways in the human brain. Neuroimage 2005;24:656-66 CrossRef Medline

12. Fridriksson JY, Bonilha L, Basilakos A, et al. Revealing the dual streams of speech processing. Proc Natl Acad Sci U S A 2016;113: 15108-13 CrossRef Medline

13. Cloutman LL. Interaction between dorsal and ventral processing streams: where, when and how? Brain Lang 2013;127:251-63 CrossRef Medline

14. Tamraz JC, Comair YG. Atlas of Regional Anatomy of the Brain Using MRI: With Functional Correlations. Berlin: Springer; 2005

15. Binkofski F, Fink GR, Geyer S, et al. Neural activity in human primary motor cortex areas $4 \mathrm{a}$ and $4 \mathrm{p}$ is modulated differentially by attention to action. J Neurophysiol 2002;88:514-19 CrossRef Medline

16. Geyer S, Ledberg A, Schleicher A, et al. Two different areas within the primary motor cortex of man. Nature 1996;382:805-07 CrossRef Medline

17. Vanderah TW, Gould DJ, Nolte J. Nolte's The Human Brain: An Introduction to its Functional Anatomy. Philadelphia: Elsevier; 2016

18. Overduin SA, Servos P. Distributed digit somatotopy in primary somatosensory cortex. Neuroimage 2004;23:462-72 CrossRef Medline

19. Watson C, Kirkcaldie M, Paxinos G. The Brain: An Introduction to Functional Neuroanatomy. Amsterdam: Elsevier; 2010 
20. Chouinard PA, Paus T. The primary motor and premotor areas of the human cerebral cortex. Neuroscientist 2006;12:143-52 CrossRef Medline

21. Geyer S, Schleicher A, Zilles K. Areas 3a, 3b, and 1 of human primary somatosensory cortex. Neuroimage 1999;10:63-83 CrossRef Medline

22. Nachev P, Kennard C, Husain M. Functional role of the supplementary and pre-supplementary motor areas. Nat Rev Neurosci 2008;9: 856-69 CrossRef Medline

23. Naidich TPY, Yousry TA. Functional neuroanatomy. In: Stippich C, ed. Clinical Functional MRI: Presurgical Functional Neuroimaging. 2nd ed. Berlin: Springer; 2015:xvi

24. Chung GH, Han YM, Jeong SH, et al. Functional heterogeneity of the supplementary motor area. AJNR Am J Neuroradiol 2005;26: 1819-23 Medline

25. Nolte J. Overview of motor system. In: Nolte J, Sundstein J. The Human Brain: An Introduction to its Functional Anatomy. 4th ed. St. Louis: Mosby;1999:441-43

26. Potgieser AR, de Jong BM, Wagemakers $M$, et al. Insights from the supplementary motor area syndrome in balancing movement initiation and inhibition. Front Hum Neurosci 2014;8:960 CrossRef Medline

27. Zilles K, Amunts K. Architecture of the cerebral cortex. In: Mai K, Paxinos G, eds. The Human Nervous System. 3rd ed. Amsterdam: Elsevier Academic Press; 2012

28. Caspers S, Amunts K, Zilles, K. Posterior parietal cortex: multimodal association cortex. In: Mai K, Paxinos G, eds. The Human Nervous System. 3rd ed. Amsterdam: Elsevier Academic Press; 2012

29. Cavanna AE, Trimble MR. The precuneus: a review of its functional anatomy and behavioural correlates. Brain 2006;129: 564-83 CrossRef Medline

30. Shomstein S. Cognitive functions of the posterior parietal cortex: top-down and bottom-up attentional control. Front Integr Neurosci 2012;6:38 CrossRef Medline

31. Culham JC, Valyear KF. Human parietal cortex in action. Curr Opin Neurobiol 2006;16:205-12 CrossRef Medline

32. Vingerhoets G. Contribution of the posterior parietal cortex in reaching, grasping, and using objects and tools. Front Psychol 2014; 5:151 CrossRef Medline

33. Seghier ML. The angular gyrus: multiple functions and multiple subdivisions. Neuroscientist 2013;19:43-61 CrossRef Medline

34. Catani M, Jones DK, ffytche DH. Perisylvian language networks of the human brain. Ann Neurol 2005;57:8-16 CrossRef Medline

35. Kamali A, Sair HI, Radmanesh A, et al. Decoding the superior parietal lobule connections of the superior longitudinal fasciculus/arcuate fasciculus in the human brain. Neuroscience 2014;277:577-83 CrossRef Medline

36. Da Costa S, van der Zwaag W, Marques JP, et al. Human primary auditory cortex follows the shape of Heschl's gyrus. J Neurosci 2011; 31:14067-75 CrossRef Medline

37. Hackett TA. Anatomic organization of the auditory cortex. Handb Clin Neurol 2015;129:27-53 CrossRef Medline

38. Poeppel D. Pure word deafness and the bilateral processing of the speech code. Cognit Sci 2001;25:679-93 CrossRef
39. Stefanatos GA, Gershkoff A, Madigan S. On pure word deafness, temporal processing, and the left hemisphere. Int Neuropsychol Soc 2005;11:456-70; discussion 455 CrossRef Medline

40. Buchsbaum BR, Baldo J, Okada K, et al. Conduction aphasia, sensorymotor integration, and phonological short-term memory: an aggregate analysis of lesion and fMRI data. Brain Lang 2011;119:119-28 CrossRef Medline

41. Tremblay P, Dick AS. Broca and Wernicke are dead, or moving past the classic model of language neurobiology. Brain Lang 2016;162: 60-71 CrossRef Medline

42. Mesulam MM, Thompson CK, Weintraub S, et al. The Wernicke conundrum and the anatomy of language comprehension in primary progressive aphasia. Brain 2015;138:2423-37 CrossRef Medline

43. Ongür D, Price JL. The organization of networks within the orbital and medial prefrontal cortex of rats, monkeys and humans. Cereb Cortex 2000;10:206-19 CrossRef Medline

44. Gasquoine PG. Localization of function in anterior cingulate cortex: from psychosurgery to functional neuroimaging. Neurosci Biobehav Rev 2013;37:340-48 CrossRef Medline

45. Leech R, Sharp DJ. The role of the posterior cingulate cortex in cognition and disease. Brain 2014;137:12-32 CrossRef Medline

46. Amiez C, Petrides M. Neuroimaging evidence of the anatomo-functional organization of the human cingulate motor areas. Cereb Cortex 2014;24:563-78 CrossRef Medline

47. Ungerleider LG, Haxby JV. 'What' and 'where' in the human brain. Curr Opin Neurobiol 1994;4:157-65 Medline

48. Kanwisher N, Yovel G. The fusiform face area: a cortical region specialized for the perception of faces. Philos Trans R Soc Lond B Biol Sci 2006;361:2109-28 CrossRef Medline

49. Kanwisher N, McDermott J, Chun MM. The fusiform face area: a module in human extrastriate cortex specialized for face perception. J Neurosci 1997;17:4302-11 Medline

50. Rossion B, Jacques C, Jonas J. Mapping face categorization in the human ventral occipitotemporal cortex with direct neural intracranial recordings. Ann N Y Acad Sci 2018 CrossRef Medline

51. Cohen L, Dehaene S. Specialization within the ventral stream: the case for the visual word form area. Neuroimage 2004;22:466-76 CrossRef Medline

52. Seghier ML, Price CJ. Explaining left lateralization for words in the ventral occipitotemporal cortex. J Neurosci 2011;31:14745-53 CrossRef Medline

53. Glezer LS, Jiang X, Riesenhuber M. Evidence for highly selective neuronal tuning to whole words in the "visual word form area". Neuron 2009;62:199-204 CrossRef Medline

54. Leff AP, Spitsyna G, Plant GT, et al. Structural anatomy of pure and hemianopic alexia. J Neurol Neurosurg Psychiatry 2006;77:1004-07 CrossRef Medline

55. Mendoza JE, Foundas AL. The cerebral cortex. Clinical neuroanatomy: a neurobehavioral approach. New York: Springer; 2008:271-499

56. Bogousslavsky J, Miklossy J, Deruaz JP, et al. Lingual and fusiform gyri in visual processing: a clinico-pathologic study of superior altitudinal hemianopia. J Neurol Neurosurg Psychiatry 1987;50:607-14 CrossRef Medline 